\title{
Necessary and Sufficient Condition for an Orthogonal Scaling Function on Vilenkin Groups
}

\section{G. S. Berdnikov}

Gleb S. Berdnikov, https://orcid.org/0000-0001-5070-4370, Saratov State University, 83 Astrakhanskaya St., 410012 Saratov, Russia, evrointelligent@gmail.com

There are several approaches to the problem of construction of an orthogonal MRA on Vilenkin groups, but all of them are reduced to the search of the so-called scaling function. In $2005 \mathrm{Yu}$. Farkov used the so-called "blocked sets" in order to find all possible band-limited scaling functions with compact support for each set of certain parameters and his conditions are necessary and sufficient. S. F. Lukomskii, lu. S. Kruss and G. S. Berdnikov presented another approach in 2014-2015 which has some advantages over the previous ones and employs the notion from discrete mathematics to achieve the same goals. This approach gives an algorithm for construction of band-limited orthogonal scaling functions with compact support in a concrete fashion using some class of directed graphs, which, in turn, is obtained from the so-called $N$-valid trees introduced by the same authors in 2012. Up to this point, though, it was not known whether this algorithm is good enough to produce any possible orthogonal scaling function of such a class. This paper describes the aforementioned algorithm and proves that it can be viewed as a necessary and sufficient condition itself, i. e. it produces any possible orthogonal scaling function. Additionally, we get another, more convenient description of the class of directed graphs we are interested in.

Keywords: Vilenkin group, abelian group, wavelets, scaling function, MRA, directed graphs .

Received: 16.10.2018 / Accepted: 18.12.2018 / Published online: 28.02.2019

DOI: https://doi.org/10.18500/1816-9791-2019-19-1-24-33

\section{INTRODUCTION}

Consider $(G, \dot{+})$ - locally compact Vilenkin group with sequences infinite in both directions as its elements:

$$
x=\left(\ldots, 0_{n-1}, x_{n}, x_{n+1}, \ldots\right), \quad x_{j}=\overline{0, p-1},
$$

where $p$ is an arbitrary prime number; $g_{n}=\left(\ldots, 0_{n-1}, 1_{n}, 0_{n+1}, \ldots\right)$ are basic elements in $G$. Addition $\dot{+}$ is defined as coordinate wise addition modulus $p$, i.e. $x \dot{+} y=\left(x_{j} \dot{+} y_{j}\right)=\left(x_{j}+y_{j} \bmod p\right)$. Let

$$
G_{n}=\left\{x \in G: x=\left(\ldots, 0_{n-1}, x_{n}, x_{n+1}, \ldots\right)\right\}, \quad n \in \mathbb{Z}
$$

be a basic sequence of subgroups, $G_{n}^{\perp}$ - sequence of ahnihilators, $X$ - character group, $r_{n} \in G_{n+1}^{\perp} \backslash G_{n}^{\perp}-$ Rademacher functions on group $G$. Dilation operator $\mathscr{A}$ in group $G$ is defined by the equation $\mathscr{A} x:=\sum_{n=-\infty}^{+\infty} a_{n} g_{n-1}$, where $x=\sum_{n=-\infty}^{+\infty} a_{n} g_{n} \in G$; in character group it is defined by the equation $(\chi \mathscr{A}, x)=(\chi, \mathscr{A} x)$. Let us define sets

$$
\begin{aligned}
H_{0}^{(s)} & =\left\{h \in G: h=a_{-1} g_{-1} \dot{+} a_{-2} g_{-2} \dot{+} \ldots \dot{+} a_{-s} g_{-s}\right\}, \quad s \in \mathbb{N}, \\
H_{0} & =\left\{x \in G: x=a_{-1} g_{-1} \dot{+} a_{-2} g_{-2} \dot{+} \ldots \dot{+} a_{-s} g_{-s}, \quad s \in \mathbb{N}\right\} .
\end{aligned}
$$

Set $H_{0}$ is the set of shifts in $G$. It is an analogue of the nonnegative integers set.

V. Protasov, Yu. Farkov in [1-3] characterized all diadic wavelets on $\mathbb{R}_{+}$and developed an algorithm for their construction. Yu. Farkov in $[4,5]$ researched scaling 
functions $\varphi(x)$ with compact support on $G_{-N}$ and developed necessary and sufficient conditions on mask $m_{0}(\chi)$, which generates an orthogonal MRA. These conditions hold with additional assumption

$$
\sum_{\alpha_{0}=0}^{p-1}\left|m_{0}\left(G_{-N}^{\perp} r_{-N}^{\alpha_{-N}} r_{-N+1}^{\alpha_{-N+1}} \ldots r_{-1}^{\alpha_{-1}} r_{0}^{\alpha_{0}}\right)\right|^{2}=1,
$$

which is necessary for orthogonality of the system of shifts of the corresponding scaling function $\varphi$. Yu. Farkov proved that in this case scaling function $\varphi$ generates orthogonal MRA iff mask $m_{0}$ does not have the so-called "blocked" sets. The problem of finding such sets requires exhaustive search of approximately $2^{p^{N}}$ different cases, which is possible only with $p$ and $N$ being rather small.

Thus, the necessity of finding another algorithm arose, the algorithm which does not require exhaustive search. This necessity triggered the appearance of another approach, which employs various graphs as the means to construct orthogonal MRA. In $[6,7]$ another algorithm for construction of $\varphi$ was developed. It doesn't require exhaustive search, but it is valid only for functions $|\hat{\varphi}(\chi)|$ constant on cosets $G_{-1}^{\perp}$ and taking 2 values only: 0 or 1 . Initially, trees appeared in $[8,9]$, where they were used for construction of Riesz MRA. In [10] authors managed to get rid of restriction $\operatorname{supp} \varphi(x) \subset G_{-1}$. To achieve this, the notion of $N$-valid tree was introduced. It was proved that step function $\varphi(x)$ with support $\operatorname{supp} \varphi(x) \subset G_{-N}$ and restriction $|\hat{\varphi}(\chi)|=0$ or 1 generates orthogonal MRA if $\varphi(x)$ is constructed by the means of some $N$-valid tree using the algorithm presented in the same paper.

In [11] another restriction was omitted. The results of this paper no longer require $\hat{\varphi}(\chi)$ to satisfy " $|\hat{\varphi}(\chi)|=0$ or 1 ". The algorithm for construction of orthogonal scaling function now has the only restriction: $\hat{\varphi}(\chi)$ is a band limited function with compact support. This algorithm does not require exhaustive search. The problem of constructing such function is reduced to constructing some digraph, which, in turn, is constructed using arbitrary $N$-valid tree.

However, until current article it was not known whether the aforementioned algorithm is able to construct any possible function $\varphi$ of the described class or not. The research presented here answers this question with definite "yes". Thus, the algorithm in question can actually be viewed as a necessary and sufficient condition for $\varphi$ to generate an orthogonal MRA on Vilenkin group. As a pleasant complement we incidentally get another, more convenient description of a certain class of digraphs while proving this fact.

The structure of the paper is the following. In Section 1 we describe the algorithm from the paper [11]. In Section 2 we find a necessary condition for scaling function $\varphi$ using the notion of digraphs, and then we prove that this necessary condition is just a rephrased sufficient one we have in the form of the algorithm, which, in turn, proves that the algorithm is a necessary and sufficient condition.

\section{CONSTRUCTION OF A SCALING FUNCTION}

Let us introduce the algorithm for construction of scaling function with the use of digraphs. Denote the collection of step functions constant on cosets of $G_{M}$ with support $\operatorname{supp}(\varphi) \subset G_{-N}$ as $\mathfrak{D}_{M}\left(G_{-N}\right), M, N \in \mathbb{N}$. Similarly, $\mathfrak{D}_{-N}\left(G_{M}^{\perp}\right)$ is a collection of step functions constant on cosets of $G_{-N}^{\perp}$ with support $\operatorname{supp}(\varphi) \subset G_{M}^{\perp}$. If $\varphi \in \mathfrak{D}_{M}\left(G_{-N}\right)$ generates an orthogonal MRA, then it satisfies the refinement equation 
$\varphi(x)=\sum_{h \in H_{0}^{(N+1)}} \beta_{h} \varphi(\mathscr{A} x \dot{-} h)$ which can also be written in frequency form (see [7])

$$
\hat{\varphi}(\chi)=m_{0}(\chi) \hat{\varphi}\left(\chi \mathscr{A}^{-1}\right)
$$

where

$$
m_{0}(\chi)=\frac{1}{p} \sum_{h \in H_{0}^{(N+1)}} \beta_{h} \overline{\left(\chi \mathscr{A}^{-1}, h\right)}
$$

is a mask of equation (1).

In [7] the following statements were proved.

1. If $\hat{\varphi}(\chi) \in \mathfrak{D}_{-N}\left(G_{M}^{\perp}\right)$ is a solution of refinement equation (1) and the system of shifts $(\varphi(x \dot{-} h))_{h \in H_{0}}$ is orthonormal, then $\varphi$ generates an orthogonal MRA.

2. If $\hat{\varphi}(\chi) \in \mathfrak{D}_{-N}\left(G_{M}^{\perp}\right)$, then the system of shifts $(\varphi(x \dot{-} h))_{h \in H_{0}}$ is orthonormal iff for all $\alpha_{-N}, \alpha_{-N+1}, \ldots, \alpha_{-1}=\overline{(0, p-1)}$

$$
\sum_{\alpha_{0}, \alpha_{1}, \ldots, \alpha_{M-1}=0}^{p-1}\left|\hat{\varphi}\left(G_{-N}^{\perp} r_{-N}^{\alpha_{-N}} \ldots r_{0}^{\alpha_{0}} \ldots r_{M-1}^{\alpha_{M-1}}\right)\right|^{2}=1
$$

Thus, in order to construct orthogonal MRA one needs to construct a function $\hat{\varphi}(\chi) \in \mathfrak{D}_{-N}\left(G_{M}^{\perp}\right)$, which is a solution of refinement equation (1) and which satisfies conditions (3).

Definition 1. Let $N$ be a natural number, $p-$ a prime number. Then $N$-valid tree is a tree with vertices $\alpha_{j}=\overline{0, p-1}$ directed from leaves to root and having the following properties:

1) the root and all vertices up to $(N-1)$-th level are equal to zero;

$2)$ any path $\left(\alpha_{k} \rightarrow \alpha_{k+1} \rightarrow \cdots \rightarrow \alpha_{k+N-1}\right)$ of length $N-1$ is unique in the tree. Here $\alpha_{i}=\overline{0, p-1}$.

Let us choose an arbitrary $N$-valid tree $T$ and construct a scaling function using it.

Algorithm 1. From the tree $T$ we construct a new tree $\tilde{T}$ in a following way.

1. Replace the path of $N$ zeros ending with root with one vertex $\left(0_{N}, 0_{N-1}, \ldots, 0_{1}\right)$. All vertices of $(N+1)$-th level of $T$ are now connected to this vertex in $\tilde{T}$. It becomes the root of $\tilde{T}$.

2. Then we change the values of each vertex without changing the arcs. If in the tree $T$ we had a path

$$
\alpha_{N} \rightarrow \alpha_{N-1} \rightarrow \cdots \rightarrow \alpha_{1}
$$

starting from the vertex $\alpha_{N}$, then in the new tree $\tilde{T}$ this vertex has a value equal to $N$-dimensional vector $\left(\alpha_{N}, \alpha_{N-1}, \ldots, \alpha_{1}\right)$.

Because of $N$-validity of the tree $T$ each possible vector appears in $\tilde{T}$ one time exactly. Also, if we denote $\operatorname{height}(T)=H$, height $(\tilde{T})=\tilde{H}$, then, obviously, $\tilde{H}=H-N+1$.

Remark. We refer to the tree $\tilde{T}$ as an expanded $N$-valid tree. Tree $T$, in turn, is called shortened $N$-valid tree. It is easy to switch from one representation to another if needed, and they describe the same structure as it becomes apparent later in this paper. 
Algorithm 2. Now we use $\tilde{T}$ to construct digraph $\Gamma$.

Each vertex $\bar{\alpha}_{N}=\left(\alpha_{N}, \alpha_{N-1}, \ldots, \alpha_{1}\right)$ of $\tilde{T}$ we can connect to any number of lower level vertices $\left(\alpha_{N-1}, \ldots, \alpha_{1}, \alpha_{0}\right)$, i.e. first $(N-1)$ elements of this vertex are equal to last $(N-1)$ elements of vertex $\bar{\alpha}_{N}$. We call this condition suffix-prefix property. Vertices that $\bar{\alpha}_{N}$ is connected to, we denote as $\left(\alpha_{N-1}, \ldots, \alpha_{1}, \tilde{\alpha}_{0}\right)$. I.e. $\alpha_{0} \in\left\{\tilde{\alpha}_{0}\right\}$ iff the vertex $\bar{\alpha}_{N}$ is connected to $\left(\alpha_{N-1}, \ldots, \alpha_{1}, \alpha_{0}\right)$ in $\Gamma$.

\section{Algorithm 3.}

1. Denote

$$
\lambda_{\alpha_{-N}, \alpha_{-N+1}, \ldots, \alpha_{-1}, \alpha_{0}}=\left|m_{0}\left(G_{-N}^{\perp} r_{-N}^{\alpha_{-N}} r_{-N+1}^{\alpha_{-N+1}} \ldots r_{-1}^{\alpha_{-1}} r_{0}^{\alpha_{0}}\right)\right|^{2} .
$$

If a vertex $\left(\alpha_{-N}, \alpha_{-N+1}, \ldots, \alpha_{-1}\right)$ in digraph $\Gamma$ is connected to vertices

$$
\left(\alpha_{-N+1}, \alpha_{-N+2}, \ldots, \alpha_{-1}, \tilde{\alpha}_{0}\right)
$$

then we define the values of the mask for the condition

$$
\sum_{\tilde{\alpha}_{0}} \lambda_{\alpha_{-N}, \alpha_{-N+1}, \ldots, \alpha_{-1}, \tilde{\alpha}_{0}}=1 \text { and } \lambda_{\alpha_{-N}, \alpha_{-N+1}, \ldots, \alpha_{-1}, \alpha_{0}}=0 \text { for all } \alpha_{0} \notin\left\{\tilde{\alpha}_{0}\right\}
$$

to hold. Also, define $m_{0}\left(G_{-N}^{\perp}\right)=1$, which implies $\lambda_{0,0, \ldots, 0}=1$.

2 . Using equation (1), one can recover $\hat{\varphi}$ from the mask we have already generated. Then the scaling function $\varphi$ itself can be found after the application of inverse Fourier transform.

The main result of paper [11] is the following.

Theorem 1. Given arbitrary $N$-valid tree $T$, let the tree $\tilde{T}$ and graph $\Gamma$ be constructed based on $T$ and values of the mask $m_{0}(\chi)$ defined with the help of equation (4). Let $\tilde{H}=$ height $(\tilde{T})$. Then the equation

$$
\hat{\varphi}(\chi)=\prod_{k=0}^{\infty} m_{0}\left(\chi \mathscr{A}^{-k}\right) \in \mathfrak{D}_{-N}\left(G_{M}^{\perp}\right)
$$

defines an orthogonal scaling function $\varphi(x) \in \mathfrak{D}_{M}\left(G_{-N}\right)$, where $M=\tilde{H}-N$.

This theorem supplied with aforementioned algorithms describes the process of constructing step scaling functions with compact support on Vilenkin groups. This process always results in an appropriate scaling function, i.e. it can be viewed as a sufficient condition. But can we acquire any possible scaling function with these properties? To answer this question some additional operations should be introduced.

\section{THE NECESSARY CONDITION AND CRITERIA}

Let us describe an algorithm inverse to Algorithm 3. Algorithm 3 describes construction of $\hat{\varphi}$ given digraph $\Gamma$, a new algorithm describes a process of construction of a digraph $\Gamma$ given $\hat{\varphi}(\chi) \in \mathfrak{D}_{-N}\left(\mathfrak{G}_{M}^{\perp}\right)$.

\section{Algorithm 4.}

1. Let vertices of our digraph $\Gamma$ be in the form $\bar{\alpha}^{j}=\left(\alpha_{i}^{j}\right)_{i=1}^{N}$. Denote $\left\{\bar{\alpha}^{j}\right\}-$ the set of all vertices.

2. Let $\hat{\varphi}\left(\mathfrak{G}_{-N}^{\perp} r_{-N}^{\alpha_{-N}} r_{-N+1}^{\alpha_{-N+1}} \ldots r_{0}^{\alpha_{0}} \ldots r_{s-1}^{\alpha_{s-1}}\right) \neq 0$, where $s \leqslant M$. Using

$$
\hat{\varphi}(\chi)=\prod_{n=0}^{\infty} m_{0}\left(\chi \mathscr{A}^{-n}\right),
$$


periodicity of the mask and notation

$$
m_{0}\left(\mathfrak{G}_{-N}^{\perp} r_{-N}^{\alpha_{-N}} r_{-N+1}^{\alpha_{-N+1}} \ldots r_{0}^{\alpha_{0}}\right)=\lambda_{\alpha_{-N}, \alpha_{-N+1}, \ldots, \alpha_{0}},
$$

we obtain:

$$
\begin{gathered}
\hat{\varphi}\left(\mathfrak{G}_{-N}^{\perp} r_{-N}^{\alpha_{-N}} r_{-N+1}^{\alpha_{-N+1}} \ldots r_{0}^{\alpha_{0}} \ldots r_{s-1}^{\alpha_{s-1}}\right)=\lambda_{\alpha_{-N}, \alpha_{-N+1}, \ldots, \alpha_{0}} \lambda_{\alpha_{-N+1}, \alpha_{-N+2}, \ldots, \alpha_{1}} \cdots \times \\
\times \lambda_{\alpha_{s-N-1}, \alpha_{s-N}, \ldots, \alpha_{s-1}} \lambda_{\alpha_{s-N}, \alpha_{s-N+1}, \ldots, \alpha_{s-1}, 0} \ldots \lambda_{\alpha_{s-1}, 0, \ldots, 0} \neq 0 .
\end{gathered}
$$

Inequality to zero holds iff all the values $\lambda_{\alpha_{i-N}, \ldots, \alpha_{i}}$, in this equation are nonequal to zero. For every such $\lambda$ we construct an arc

$$
\left(\alpha_{i-N}, \alpha_{i-N+1} \ldots, \alpha_{i-1}\right) \rightarrow\left(\alpha_{i-N+1}, \alpha_{i-N+2}, \ldots, \alpha_{i}\right) .
$$

3. Checking every coset for each $\hat{\varphi}(\chi)$ is nonequal to zero and performing the same operations we obtain digraph $\Gamma$ where each arc corresponds to a different nonzero value of the mask.

Theorem 2 (Necessary condition in terms of graphs). Let $\varphi(x)$ be a scaling function with $\hat{\varphi}(\chi) \in \mathfrak{D}_{-N}\left(\mathfrak{G}_{M}^{\perp}\right)$ which generates orthogonal MRA on Vilenkin group. Then digraph $\Gamma$ constructed with the algorithm 4 has the following properties:

1. If there exists an arc $\bar{\alpha}^{j} \rightarrow \bar{\alpha}^{k}$, it means that $N-1$ last components of $\bar{\alpha}^{j}$ coincide with the first $N-1$ components of $\bar{\alpha}^{k}$. In other words, suffix-prefix condition holds.

2. There exists a path to $\overline{0}=(0,0, \ldots, 0)$ from any vertex that is not $\overline{0}$.

3. There are no directed cycles in the graph.

4. The vertex $\overline{0}$ is a source, i.e. there are no arcs coming out of it.

5. The graph includes all possible vertices $\left(\alpha_{-N}, \alpha_{-N+1}, \ldots, \alpha_{-1}\right), \forall \alpha_{i}=\overline{0, p-1}$.

Proof. 1. This property is apparent by the construction algorithm.

2. Let us prove that there exists a path from any nonzero vertex to $\overline{0}=(0,0, \ldots, 0)$. Since all cosets from the support of $\hat{\varphi}(\chi)$ have the form $\mathfrak{G}_{-N}^{\perp} r_{-N}^{\alpha_{-N}} r_{-N+1}^{\alpha_{-N+1}} \ldots r_{0}^{\alpha_{0}} \ldots r_{s-1}^{\alpha_{s-1}}$ and

$$
\begin{gathered}
\hat{\varphi}\left(\mathfrak{G}_{-N}^{\perp} r_{-N}^{\alpha_{-N}} r_{-N+1}^{\alpha_{-N+1}} \ldots r_{0}^{\alpha_{0}} \ldots r_{s-1}^{\alpha_{s-1}}\right)=\lambda_{\alpha_{-N}, \alpha_{-N+1}, \ldots, \alpha_{0}} \lambda_{\alpha_{-N+1}, \alpha_{-N+2}, \ldots, \alpha_{1}} \cdots \times \\
\times \lambda_{\alpha_{s-N-1}, \alpha_{s-N}, \ldots, \alpha_{s-1}} \lambda_{\alpha_{s-N}, \alpha_{s-N+1}, \ldots, \alpha_{s-1}, 0} \ldots \lambda_{\alpha_{s-1}, 0, \ldots, 0} \neq 0 .
\end{gathered}
$$

all values of $\lambda$ in this product are nonzero. This collection of values of $\lambda$ generates a path

$$
\left(\alpha_{-N}, \alpha_{-N+1}, \ldots, \alpha_{1}\right) \rightarrow\left(\alpha_{-N+1}, \alpha_{-N+2}, \ldots, \alpha_{0}\right) \rightarrow \cdots \rightarrow\left(\alpha_{s}, 0, \ldots, 0\right) \rightarrow(0,0, \ldots, 0) .
$$

Thus, since any coset from the support of $\hat{\varphi}(\chi)$ generates a path ending with $\overline{0}$, there exists a path from any nonzero vertex to $\overline{0}=(0,0, \ldots, 0)$. This proves the property.

3 . Let us prove this one by contradiction. Let the graph $\Gamma$ contain a directed cycle

$$
\bar{\alpha}^{1} \rightarrow \bar{\alpha}^{2} \rightarrow \ldots \bar{\alpha}^{k} \rightarrow \bar{\alpha}^{1}
$$

We rewrite this path using the 1st property and specifying the components of vertices:

$$
\left(\alpha_{1}, \alpha_{2}, \ldots, \alpha_{N-1}, \alpha_{N}\right) \rightarrow\left(\alpha_{2}, \alpha_{3}, \ldots, \alpha_{N}, \alpha_{N+1}\right) \rightarrow \ldots
$$




$$
\begin{aligned}
\cdots \rightarrow & \left(\alpha_{k-N+1}, \alpha_{k-N}, \ldots, \alpha_{k-1}, \alpha_{k}\right) \rightarrow\left(\alpha_{k-N+2}, \alpha_{k-N+3}, \ldots, \alpha_{k}, \alpha_{1}\right) \rightarrow \\
& \rightarrow \cdots \rightarrow\left(\alpha_{k}, \alpha_{1}, \ldots, \alpha_{N-1}, \alpha_{N}\right) \rightarrow\left(\alpha_{1}, \alpha_{2}, \ldots, \alpha_{N-1}, \alpha_{N}\right) .
\end{aligned}
$$

By construction, every arc corresponds to nonzero value of $\lambda$. Existence of such path means that

$$
\lambda_{\alpha_{1}, \alpha_{2}, \ldots, \alpha_{N}, \alpha_{N+1}} \lambda_{\alpha_{2}, \alpha_{3}, \ldots, \alpha_{N+1}, \alpha_{N+2}} \ldots \lambda_{\alpha_{k}, \alpha_{1}, \ldots, \alpha_{N-1}, \alpha_{N}} \neq 0 .
$$

Let us recall that by the 2 nd property there exists a path from any vertex to $\overline{0}$ vertex. Thus, at least one vertex $\bar{\alpha}^{j}$ from the cycle is connected not only to $\bar{\alpha}^{j+1}$, but is also a part of a path $\bar{\alpha}^{j} \rightarrow \bar{\alpha}^{l} \rightarrow \bar{\alpha}^{l+1} \rightarrow \cdots \rightarrow \overline{0}$.

Without the loss of generality we consider this vertex to be $\bar{\alpha}^{1}$. Consequently, there exists a path

$$
\begin{gathered}
\left(\alpha_{1}, \alpha_{2}, \ldots, \alpha_{N-1}, \alpha_{N}\right) \rightarrow\left(\alpha_{2}, \alpha_{3}, \ldots, \alpha_{N}, \alpha_{l}\right) \rightarrow\left(\alpha_{2}, \alpha_{3}, \ldots, \alpha_{l}, \alpha_{l+1}\right) \rightarrow \cdots \rightarrow \\
\rightarrow\left(\alpha_{s-N}, \alpha_{s-N+1}, \ldots, \alpha_{s-1}, \alpha_{s}\right) \rightarrow\left(\alpha_{s-N+1}, \alpha_{s-N+2}, \ldots, \alpha_{s}, 0\right) \rightarrow \ldots \\
\cdots \rightarrow\left(\alpha_{s}, 0, \ldots, 0,0\right) \rightarrow \overline{0}
\end{gathered}
$$

which corresponds to the product

$$
\lambda_{\alpha_{1}, \alpha_{2}, \ldots, \alpha_{N}, \alpha_{l}} \lambda_{\alpha_{2}, \alpha_{3}, \ldots, \alpha_{l}, \alpha_{l+1}} \ldots \lambda_{\alpha_{s-N-1}, \alpha_{s-N}, \ldots, \alpha_{s-1}} \lambda_{\alpha_{s-N}, \alpha_{s-N+1}, \ldots, \alpha_{s-1}, 0} \ldots \lambda_{\alpha_{s-1}, 0, \ldots, 0} \neq 0 .
$$

It is possible to construct a product

$$
\begin{aligned}
& \lambda_{\alpha_{1}, \alpha_{2}, \ldots, \alpha_{N}, \alpha_{N+1}} \lambda_{\alpha_{2}, \alpha_{3}, \ldots, \alpha_{N+1}, \alpha_{N+2}} \ldots \lambda_{\alpha_{k}, \alpha_{1}, \ldots, \alpha_{N-1}, \alpha_{N}} \times \\
& \times \lambda_{\alpha_{1}, \alpha_{2}, \ldots, \alpha_{N}, \alpha_{N+1}} \lambda_{\alpha_{2}, \alpha_{3}, \ldots, \alpha_{N+1}, \alpha_{N+2}} \ldots \lambda_{\alpha_{k}, \alpha_{1}, \ldots, \alpha_{N-1}, \alpha_{N}} \times \\
& \times \lambda_{\alpha_{1}, \alpha_{2}, \ldots, \alpha_{N}, \alpha_{N+1}} \lambda_{\alpha_{2}, \alpha_{3}, \ldots, \alpha_{N+1}, \alpha_{N+2}} \ldots \lambda_{\alpha_{k}, \alpha_{1}, \ldots, \alpha_{N-1}, \alpha_{N}} \times \\
& \times \lambda_{\alpha_{1}, \alpha_{2}, \ldots, \alpha_{N}, \alpha_{l}} \lambda_{\alpha_{2}, \alpha_{3}, \ldots, \alpha_{l}, \alpha_{l+1}} \ldots \lambda_{\alpha_{s-N-1}, \alpha_{s-N}, \ldots, \alpha_{s-1}} \lambda_{\alpha_{s-N}, \alpha_{s-N+1}, \ldots, \alpha_{s-1}, 0} \ldots \lambda_{\alpha_{s-1}, 0, \ldots, 0} \neq 0,
\end{aligned}
$$

where the product $\lambda_{\alpha_{1}, \alpha_{2}, \ldots, \alpha_{N}, \alpha_{N+1}} \lambda_{\alpha_{2}, \alpha_{3}, \ldots, \alpha_{N+1}, \alpha_{N+2}} \ldots \lambda_{\alpha_{k}, \alpha_{1}, \ldots, \alpha_{N-1}, \alpha_{N}}$ is multiplied by itself $n$ times, $n$ is an arbitrary natural number.

This product means that $\forall n \in \mathbb{N}$ the following is true:

$$
\hat{\varphi}\left(\mathfrak{G}_{-N}^{\perp} \prod_{i=0}^{n-1}\left(r_{-N+i k}^{\alpha_{1}} r_{-N+i k+1}^{\alpha_{2}} \ldots r_{-N+i k+k-1}^{\alpha_{k}}\right) \times r_{-N+n k}^{\alpha_{l}} r_{-N+n k+1}^{\alpha_{l+1}} \ldots r_{-N+n k+s-l}^{\alpha_{s}}\right) \neq 0 .
$$

This contradicts the compactness of support of $\hat{\varphi}(\chi)$. Thus, $\Gamma$ does not contain directed cycles.

4. This property follows from the second and the third ones. We also prove it by contradiction.

Let an $\operatorname{arc} \overline{0} \rightarrow \bar{\alpha}^{j}$ exist in $\Gamma$. By the second property there exists a path $\bar{\alpha}^{j} \rightarrow \cdots \rightarrow \overline{0}$. Thus there exists a directed cycle $\overline{0} \rightarrow \bar{\alpha}^{j} \rightarrow \cdots \rightarrow \overline{0}$, which contradicts the third property.

5. Since the necessary condition of the mask $\sum_{\alpha_{0}}\left|\lambda_{\alpha_{-N}, \alpha_{-N+1}, \ldots, \alpha_{-1}, \alpha_{0}}\right|^{2}=1$ holds, for any collection $\alpha_{-N}, \alpha_{-N+1}, \ldots, \alpha_{-1}$ there exists at least one $\alpha_{0}$ such that the 
corresponding value of the mask is not equal to 0 . This, in turn, means that for every such collection there exists an arc

$$
\left(\alpha_{-N}, \alpha_{-N+1}, \ldots, \alpha_{-1}\right) \rightarrow\left(\alpha_{-N+1}, \ldots, \alpha_{-1}, \alpha_{0}\right) .
$$

Thus, the constructed graph includes all possible vertices $\left(\alpha_{-N}, \alpha_{-N+1}, \ldots, \alpha_{-1}\right)$, $\forall \alpha_{i}=\overline{0, p-1}$.

Let us denote the collection of graphs constructed using algorithms $1-2$ as $\boldsymbol{\Gamma}_{\mathbf{1}}$. Let us denote the collection of graphs satisfying the properties of Theorem 2 as $\boldsymbol{\Gamma}_{\mathbf{2}}$. At this point we can see the following implication:

$$
\begin{aligned}
& \Gamma \in \Gamma_{1} \text { generates } \hat{\varphi}-\mathrm{FT} \text { of a scaling function } \Rightarrow \\
& \Rightarrow \hat{\varphi}-\mathrm{FT} \text { of a scaling function-generates } \Gamma \in \Gamma_{\mathbf{2}},
\end{aligned}
$$

thus $\Gamma_{1} \subset \Gamma_{2}$. But if the set $\Gamma_{2}$ is wider then the algorithms 1-3 are not able to generate any possible scaling function! On the other hand, if $\boldsymbol{\Gamma}_{1}=\boldsymbol{\Gamma}_{2}$ then the algorithms are actually the necessary and sufficient condition for $\hat{\varphi}$ to be an orthogonal scaling function with compact support. Let us prove this.

Theorem 3. Set $\Gamma_{1}$ contains the set $\Gamma_{2}$, i.e. $\Gamma_{2} \subset \Gamma_{1}$.

Proof. To prove the fact, we need to show that any graph satisfying the properties from Theorem 2 can be constructed from some $N$-valid tree using algorithms 1-2.

Consider $\Gamma \in \Gamma_{\mathbf{2}}$. Let us construct $\tilde{T}$ from algorithm 1 based on this graph.

Step 1. Choose $\bar{\alpha}^{1}=\left(\alpha_{1}^{1}, \alpha_{2}^{1}, \ldots, \alpha_{N}^{1}\right), \alpha_{i}^{1}=\overline{0, p-1}$. Then choose the longest path of the form $p^{(1)}=\bar{\alpha}^{1} \rightarrow p^{2,(1)} \rightarrow \cdots \rightarrow \bar{p}^{l_{1},(1)} \rightarrow \overline{0}$, i. e. the longest path starting from $\bar{\alpha}^{1}$ and ending with $\overline{0}$. This path exists since there are no cycles (and thus no paths of unlimited length) and since there exists a path from each nonzero vertex to $\overline{0}$. If there exist several paths of maximal length we choose any of them. Let us include this path into the tree. At this point the tree consists of the only "branch":

$$
\bar{\alpha}^{1} \rightarrow p^{2,(1)} \rightarrow \cdots \rightarrow \bar{p}^{l_{1},(1)} \rightarrow \overline{0} .
$$

Denote $p^{1,(1)}=\bar{\alpha}^{1}, p^{l_{1}+1,(1)}=\overline{0}$.

Step 2. Choose the next value $\bar{\alpha}^{2}=\left(\alpha_{1}^{2}, \alpha_{2}^{2}, \ldots, \alpha_{N}^{2}\right), \alpha_{i}^{2}=\overline{0, p-1}$. Choose the longest path $p^{(2)}=\bar{\alpha}^{2} \rightarrow p^{2,(2)} \rightarrow \cdots \rightarrow p^{l_{2},(2)} \rightarrow \overline{0}$. Denote $p^{1,(2)}=\bar{\alpha}^{2}, p^{l_{2}+1,(2)}=\overline{0}$. Again, let us mention that if there exist several longest paths we choose an arbitrary one. Include this path into a tree.

Out of all vertices of $p^{(2)}$ already in the tree choose vertex $p^{k,(2)}$ with the lowest $k$. It's guaranteed that at least $\overline{0}$ is already in the tree. Two cases are possible: either the whole "tail" of the path $p^{k,(2)} \rightarrow p^{k+1,(2)} \rightarrow \cdots \rightarrow p^{l_{2},(2)} \rightarrow \overline{0}$ is already in the tree or not. In the latter case it means that there exists path of the same length from $p^{k,(2)}$ to $\overline{0}$. Indeed, since we chose the longest paths from $\bar{\alpha}^{2}$, the "tail" of such path starting from any $p^{m,(2)}$ is the longest path from the vertex $p^{m,(2)}$.

Case 1. The "tail" is in the tree already.

In this case $\exists k, j: p^{k,(2)}=p^{j,(1)}$, where $k, j$ are the greatest of all such values, and $\forall i, p^{k+i,(2)}=p^{j+i,(1)}$. If $k=1$ then the whole path $p^{(2)}$ is in the tree already and we do not change it. Otherwise connect the path $\bar{\alpha}^{2} \rightarrow \cdots \rightarrow p^{k-1,(2)}$ to $p^{k,(2)}=p^{j,(1)}$. We have included his path in the tree. No cycles have appeared in the process, so our structure is a tree, still. 
Case 2. The "tail" is not in the tree.

As we have already discussed, in this case the path from $p^{k,(2)}$ to $\overline{0}$ existing in the tree (denote it by $p^{k,(2)} \rightarrow \tilde{p}^{k+1,(2)} \rightarrow \cdots \rightarrow \tilde{p}^{l_{2},(2)} \rightarrow \overline{0}$ ), has the same length as the "tail" $p^{k,(2)} \rightarrow p^{k+1,(2)} \rightarrow \cdots \rightarrow p^{l_{2},(2)} \rightarrow \overline{0}$. It means that original graph $\Gamma$ contains both of them. Let us choose

$$
\tilde{p}^{(2)}=\bar{\alpha}^{2} \rightarrow p^{2,(2)} \rightarrow \ldots p^{k,(2)} \rightarrow \tilde{p}^{k+1,(2)} \rightarrow \cdots \rightarrow \tilde{p}^{l_{2,(2)}} \rightarrow \overline{0}
$$

instead of $p^{(2)}$ and include it in the same way as Case 1.

Step $n$. Choose the next vertex $\bar{\alpha}^{n}$. Choose the longest path $p^{(n)}=\bar{\alpha}^{n} \rightarrow$ $\rightarrow p^{2,(n)} \rightarrow \ldots \rightarrow p^{l_{n},(n)} \rightarrow \overline{0}$. Assume all paths $p^{(1)}, p^{(2)}, \ldots, p^{(n-1)}$ have already been included in the tree, and include $p^{(n)}$ into it. Denote $p^{1,(i)}=\bar{\alpha}^{i}, p^{l_{i}+1,(i)}=\overline{0}$

Similarly to Step 2 we have already chosen $p^{k,(n)}$ in the tree and having the lowest possible index $k$. The "tail" of $p^{k,(n)} \rightarrow p^{k+1,(n)} \rightarrow \cdots \rightarrow p^{l_{n},(n)} \rightarrow \overline{0}$ is either in the tree already or we can rechoose it to satisfy this property.

If $k=1$ then the whole path $p^{(n)}$ is in the tree already and we don't change anything. Otherwise connect the path $\bar{\alpha}^{n} \rightarrow \cdots \rightarrow p^{k-1,(n)}$ to $p^{k,(n)}$ which is in the tree already. The path is included, no cycles have appeared, we still have a tree.

Choosing all possible $\alpha_{i}$ we obtain a tree with all those vertices. During the construction process we used paths from $\Gamma \in \Gamma_{2}$, thus:

1) every possible $\alpha_{i}$ appears in the tree only once;

2) the vertex $\overline{0}$ is a root;

3) if there exists an $\operatorname{arc} \bar{\alpha}^{i} \rightarrow \bar{\alpha}_{i+1}$ that implies that the last $N-1$ components of $\bar{\alpha}^{i}$ are equal to the first $N-1$ components of $\bar{\alpha}^{i+1}$, i.e. suffix-prefix property holds.

These properties mean that we got an expanded $N$-valid tree $\tilde{T}$. It is easy to return to the shortened one $T$ if needed. Let us now reconstruct $\Gamma$ using this tree.

All the arcs of $\tilde{T}$ also exist in $\Gamma$. Let us add the arcs which exist in $\Gamma$ but not in $\tilde{T}$.

Let us prove that each added arc is the arc from higher level vertex to the lower level one. Indeed, by construction any path $\bar{\alpha}^{k} \rightarrow \bar{\alpha}^{k+1} \rightarrow \cdots \rightarrow \bar{\alpha}^{k+s} \rightarrow \overline{0}$ of length $s+2$ is the longest path of $\Gamma$ starting from $\bar{\alpha}^{k}$. Let $\bar{\alpha}^{j}$ be a vertex of a greater or equal level, i.e. the path $\bar{\alpha}^{j} \rightarrow \bar{\alpha}^{j+1} \rightarrow \cdots \rightarrow \bar{\alpha}^{j+s_{1}} \rightarrow \overline{0}$ has the length $s_{1}+2$, where $s_{1} \geqslant s$. If we need to connect $\bar{\alpha}^{k}$ with $\bar{\alpha}^{j}$ while reconstructing arcs of $\Gamma$, it means that there exists the path $\bar{\alpha}^{k} \rightarrow \bar{\alpha}^{j} \rightarrow \bar{\alpha}^{j+1} \rightarrow \cdots \rightarrow \bar{\alpha}^{j+s_{1}} \rightarrow \overline{0}$ and it has the length of $s_{1}+3>s+2$ greater than the chosen longest path $\bar{\alpha}^{k} \rightarrow \bar{\alpha}^{k+1} \rightarrow \cdots \rightarrow \bar{\alpha}^{k+s} \rightarrow \overline{0}$. It is impossible by construction.

Thus, for an arbitrary $\Gamma \in \Gamma_{\mathbf{2}}$ we found $N$-valid tree $\tilde{T}$ which can generate $\Gamma$ using algorithm 2. The theorem is proved.

The collection of Theorems 1-3 shows that there is a bijection between all possible supports of scaling function and graphs of class $\boldsymbol{\Gamma}_{1}=\boldsymbol{\Gamma}_{2}$. Algorithms 1, 2 describe a way of constructing such graphs, algorithm 3 shows how to construct an orthogonal scaling function. On the other hand, we have a descriptions of all these graphs in Theorem 2 . These are all small, but pleasant consequences of the work. The main achievement is the following, though.

We have proven that by the use of algorithms 1-3 we can obtain any possible $\varphi(x) \in \mathfrak{D}_{M}\left(G_{-N}\right)$ which generates an orthogonal MRA on Vilenkin groups, thus the algorithms 1-3 can be viewed as a necessary and sufficient condition for such function.

Acknowledgements: This work was supported by the Russian Foundation for Basic Research (project no. 16-01-00152a). 


\section{References}

1. Protasov V. Yu., Farkov Yu. A. Dyadic wavelets and refinable functions on a half-line. Sb. Math., 2006, vol. 197, iss. 10, pp. 1529-1598. DOI: https://doi.org/10.1070/SM2006v197n10ABEH003811

2. Farkov Yu. A. Biorthogonal dyadic wavelets on $\mathbb{R}_{+}$. Russian Math. Surveys, 2007, vol. 62, iss. 6, pp. 1197-1198. DOI: https://doi.org/10.1070/RM2007v062n06ABEH004494

3. Protasov V. Yu. Dyadic wavelet approximaion. Sb. Math., 2007, vol. 198, iss. 11, pp. 16651681. DOI: https://doi.org/10.1070/SM2007v198n11ABEH003900

4. Farkov Yu. A. Orthogonal wavelets with compact support on locally compact Abelian groups. Izv. Math., 2005, vol. 69, iss. 3, pp. 623-650. DOI: https://doi.org/10.1070/IM2005v069n03ABEH000540

5. Farkov Yu. A. Orthogonal wavelets on direct products of cyclic groups. Math. Notes, 2007, vol. 82 , iss. 5 , pp. $843-859$.

6. Lukomskii S. F. Multiresolution analysis on zero-dimensional Abelian groups and wavelets bases. Sb. Math., 2010, vol. 201, iss. 5, pp. 669-691. DOI: https://doi.org/10.1070/SM2010v201n05ABEH004088

7. Lukomskii S. F. Step refinable functions and orthogonal MRA on Vilenkin groups. J. Fourier Anal. Appl., 2014, vol. 20, iss. 1, pp. 42-65. DOI: https://doi.org/10.1007/s00041-013-9301-6

8. Lukomskii S. F. Riesz Multiresolution Analysis on Vilenkin Groups. Dokl. Math., 2014, vol. 90, iss. 1, pp. 412-415. DOI: https://doi.org/10.1134/S1064562414040061

9. Lukomskii S. F. Riesz multiresolution analysis on zero-dimensional groups. Izv. Math., 2015, vol. 79, iss. 1, pp. 145-176. DOI: https://doi.org/10.1070/ IM2015v079n01ABEH002737

10. Lukomskii S. F., Berdnikov G. S. N-Valid trees in wavelet theory on Vilenkin groups. Int. J. Wavelets Multiresolut. Inf. Process, 2015, vol. 13, no. 05, 1550037. DOI: https://doi.org/10.1142/S021969131550037X

11. Berdnikov G. S., Lukomskii S. F., Kruss Iu. S. On orthogonal systems of shifts of scaling function on local fields of positive characteristic. Turk. J. Math., 2017, vol. 41, pp. 244-253. DOI: https://doi.org/10.3906/mat-1504-7

\section{Cite this article as:}

Berdnikov G. S. Necessary and Sufficient Condition for an Orthogonal Scaling Function on Vilenkin Groups. Izv. Saratov Univ. (N. S.), Ser. Math. Mech. Inform., 2019, vol. 19, iss. 1, pp. 24-33. DOI: https://doi.org/10.18500/1816-9791-2019-19-1-24-33

\section{УДК 517.986.62}

\section{Необходимое и достаточное условие ортогональной масштабирующей функции на группах Виленкина}

\section{Г. С. Бердников}

Бердников Глеб Сергеевич, ассистент касредры математического анализа, Саратовский национальный исследовательский государственный университет имени Н. Г. Чернышевского, Россия, 410012, Саратов, ул. Астраханская, д. 83, evrointelligent@gmail.com

Существуют несколько подходов к задаче построения ортогонального кратномасштабного анализа на группах Виленкина, но все они сводятся к поиску так называемой масштабирующей фрункции. В 2005 г. Ю. А. Фарков использовал так называемые «блокированные» множества, чтобы строить все возможные масштабирующие фрункции с компактным носителем и ограниченной частотной полосой 
для каждого набора неких параметров, его условия оказались необходимыми и достаточными. С. Ф. Лукомский, Ю. С. Крусс и Г. С. Бердников представили другой подход в 2014-2015 гг., который имеет некие преимущества перед другими и использует аппарат дискретной математики для достижения тех же целей. Результатом этого подхода является алгоритм построения ортогональных масштабирующих фрункций с ограниченной частотной полосой и компактным носителем в конкретном виде, используя некий класс ориентированных грасров, которые, в свою очередь, строятся по так называемым $N$-валидным деревьям, введенным теми же авторами в 2012 г. До этого момента, однако, было неизвестно, достаточно ли этот алгоритм хорош, чтобы порождать любую из возможных ортогональных масштабирующих фрункций такого класса. Эта работа описывает вышеупомянутый алгоритм и доказывает, что его можно воспринимать как необходимое и достаточное условие, то есть он может порождать любую возможную ортогональную масштабирующую фрункцию. Дополнительно мы получим другое, более удобное описание интересующего нас класса ориентированных грасров.

Ключевые слова: группа Виленкина, абелева группа, вейвлеты, масштабирующая фрункция, КМА, ориентированные грасры.

Поступила в редакцию: 16.10.2018 / Принята: 18.12 .2018 / Опубликована онлайн: 28.02.2019

Благодарности. Работа выполнена при финансовой поддержке РФФИ (проект № 16-01-00152a).

\section{Образец для цитирования:}

Berdnikov G. S. Necessary and Sufficient Condition for an Orthogonal Scaling Function on Vilenkin Groups [Бердников Г. С. Необходимое и достаточное условие ортогональной масштабирующей функции на группах Виленкина] // Изв. Сарат. ун-та. Нов. сер. Сер. Математика. Механика. Информатика. 2019. Т. 19, вып. 1. C. 24-33. DOI: https://doi.org/10.18500/18169791-2019-19-1-24-33 